\title{
Effectiveness of Autologous Platelet-Rich Plasma for the Healing of Ulcers after Endoscopic Submucosal Dissection
}

\author{
Eunju Jeong ${ }^{1 *}$, In kyung Yoo ${ }^{1^{*}}$, Ozlem Ozer Cakir ${ }^{2}$, Hee Kyung Kim ${ }^{1}$, Won Hee Kim${ }^{1}$, Sung Pyo Hong ${ }^{1}$ and Joo Young Cho ${ }^{1}$ \\ ${ }^{1}$ Digestive Disease Center, CHA Bundang Medical Center, CHA University, Seongnam, Korea, ${ }^{2}$ Alanya Alaaddin Keykubat University, School \\ of Medicine Department of Gastroenterology and Hepatology, Antalya, Turkey
}

Background/Aims: Platelet-rich plasma (PRP) has been used for wound healing in various medical fields. The aim of this study was to evaluate the clinical efficacy and safety of local PRP injections after endoscopic submucosal dissection (ESD).

Methods: Patients were non-randomly divided into the following two groups: (1) control group in which patients were administered only an intravenous proton pump inhibitor (PPI), and (2) a study group in which patients were administered an intravenous PPI and a topical PRP injection. We assessed the reduction in the ulcer area and stage of the ulcer after the procedure ( 24 hours, 48 hours, and 28 days after endoscopic surgery).

Results: We enrolled 7 study and 7 control patients. In the study group, the rate of ulcer reduction was $59 \%$ compared to $52 \%$ in the control group ( $p=0.372$ ), 28 days after ESD. There were 5 patients in the S stage and 2 patients in the $\mathrm{H}$ stage in the study group compared to no patient in the S stage and 7 patients in the $\mathrm{H}$ stage in the control group $(p=0.05), 28$ days after ESD. There were no serious complications in either group.

Conclusions: The local injection of PRP is a safe and effective procedure for ulcer healing after ESD. Clin Endosc 2019;52:472-478

Key Words: Endoscopic submucosal dissection; Platelet-rich plasma; Ulcer healing

\section{INTRODUCTION}

Endoscopic submucosal dissections (ESDs) are commonly performed for early gastric cancers (EGCs) and dysplasia, and various complications, such as hemorrhage, perforation, and stenosis, have been reported after ulcer healing. ${ }^{1-3}$ ESD causes an iatrogenic ulcer in the resection area, resulting in bleeding and abdominal pain, similar to peptic ulcers. The optimum regimen and duration of treatment for ESD-induced ulcers

Received: August 31, 2018 Revised: January 16, 2019

Accepted: January17, 2019

Correspondence: Joo Young Cho

Digestive Disease Center, CHA Bundang Medical Center, CHA University, 59 Yatap-ro, Bundang-gu, Seongnam 13496, Korea

Tel: +82-31-780-5641, Fax: +82-32-780-5005, E-mail: cjy6695@dreamwiz.com ORCID: https://orcid.org/0000-0001-9085-0313

*These authors equally contributed to this work.

(cc) This is an Open Access article distributed under the terms of the Creative Commons Attribution Non-Commercial License (http://creativecommons.org/ licenses/by-nc/3.0) which permits unrestricted non-commercial use, distribution, and reproduction in any medium, provided the original work is properly cited. remain poorly researched. ${ }^{4}$ However, recently, a proton pump inhibitor (PPI) (or $\mathrm{H} 2$ receptor blocker) has been administered empirically for $4-8$ weeks. ${ }^{5.7}$ Several studies have been conducted using polyglycolic acid adhesives, fibrin glue, and local steroid injection to prevent post-ESD complications and stenosis after ulcer healing. However, a large-scale prospective study has not been conducted to date. .10 $^{8-10}$

Interestingly, platelets are known to be involved in gastric ulcer healing, and results from animal studies show that oral administration of platelet-rich plasma (PRP) resulted in a faster recovery of gastric ulcer wounds. ${ }^{11}$ Platelets were originally only thought to play a role in the process of blood clotting, but it has been shown that they also aid tissue regeneration and healing through the action of abundant growth factors and cytokines, such as platelet-derived growth factor-AB (PDGF$\mathrm{AB})$ or transforming growth factor beta-1. ${ }^{12}$ For instance, angiogenesis is an important process in gastric ulcer healing. Various pro-angiogenic factors, including vascular endothelial growth factor (VEGF), basic fibroblast growth factor and 
PDGF, are stored in platelets. During the hemostasis phase of ulcer healing, these growth factors are produced and secreted in the new blood vessels, ${ }^{13-15}$ suggesting that they also play a role in wound healing. PRP is obtained by centrifugation of the autologous blood and contains a higher concentration of platelets than in the entire blood volume. Specifically, PRP contains more than four times the platelet concentration of normal peripheral blood. ${ }^{11}$ Currently, PRP is used in various fields such as plastic surgery, orthopedics, dermatology, and dentistry, ${ }^{16-25}$ but not in the field of gastroenterology. Thus, the aim of this study was to evaluate the clinical efficacy and safety of local PRP injections after ESD.

\section{MATERIALS AND METHODS}

\section{Study design}

This study was a non-randomized pilot study conducted from May 2017 to November 2017. Patients were divided into a control group and a study group: (1) patients in the control group only received an intravenous PPI after ESD, and (2) patients in the study group received intravenous PPI and a local PRP injection after ESD. All participants were administered a PPI intravenously for the first 3 days, followed by oral PPI administration for up to 4 weeks after the procedure.

We assessed healing of the ulcer after ESD by evaluating the ulcer size and stage. The initial ulcer size was measured by the size of the specimen after ESD. At follow-up, the ulcer size was measured by an upper gastrointestinal endoscopic probe. The Sakita and Fukutomi classification system was used to assess the ulcer stage, ${ }^{26}$ and an endoscopist scored the ulcer stage from 1 to $6(1,2$ : active ulcer [A1, 2]; 3, 4: healing ulcer [H1, 2]; 5, 6: scarring ulcer [S1, 2]).

\section{PRP preparation}

On the day of the procedure, $15 \mathrm{~mL}$ of the patient's peripheral blood was collected and mixed with $2 \mathrm{~mL}$ sodium citrate. The blood was then centrifuged at 3,500 rpm for 6 minutes. Two milliliters of PRP containing a buffy were obtained and stored in a separate bottle after centrifugation (Fig. 1). After this, $1 \mathrm{~mL}$ of PRP without additional dilution was injected into the submucosal layer in all 4 directions around the resection site (total $4 \mathrm{~mL}$ ) (Fig. 2).

\section{Patients}

Patients aged 20 to 65 years who underwent ESD for EGC and dysplasia were included in this study.

Exclusion criteria were as follows:

(1) Patients who refused to provide consent for the study;

(2) Severe or uncontrolled heart, lung, or mental ailments, and acute severe infections;

(3) History of esophageal and stomach surgery;

(4) Patients with an Eastern Cooperative Oncology Group performance status of 2 or greater;

(5) Patients without adequate bone marrow function, i.e., those with an absolute neutrophil count of $1,500 / \mathrm{mm}^{3}$ and platelets $<100,000 / \mathrm{mm}^{3}$;

(6) Creatinine levels 1.5-times above the upper limit, in patients with inadequate renal function;

(7) Aspartate transaminase and alanine aminotransferase levels 2.5 times above the normal upper limit and total bilirubin 1.5 times above the normal upper limit;

(8) Clinically-proven thrombocytopenia, or platelet dysfunction;

(9) Use of nonsteroidal anti-inflammatory drugs (NSAIDs) within 48 hours prior to the procedure;

(10) Use of systemic steroids within 2 weeks prior to the procedure;
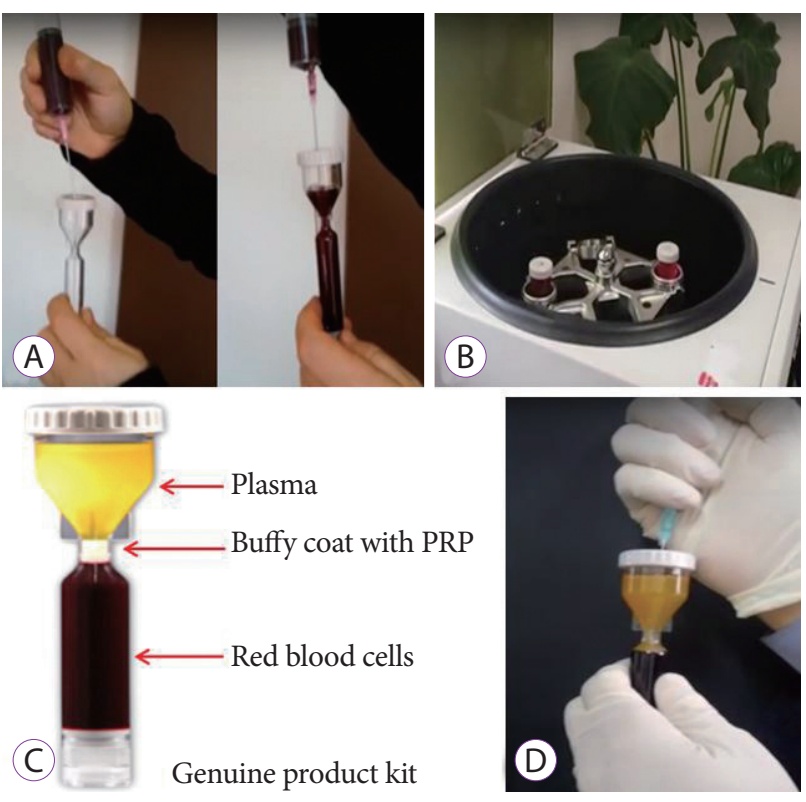

Fig. 1. Platelet-rich plasma (PRP) preparation.
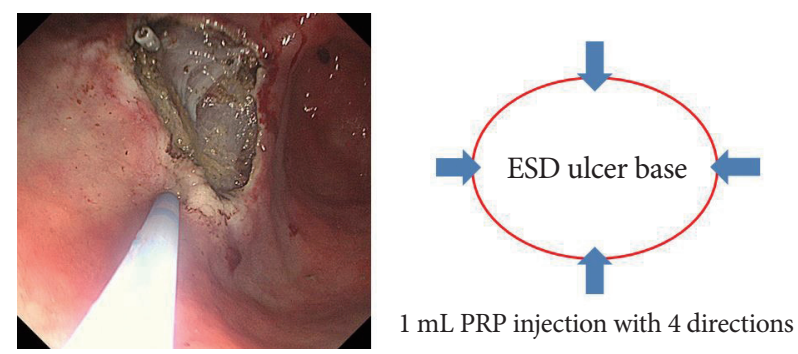

Fig. 2. Method of platelet-rich plasma (PRP) injection. ESD, endoscopic submucosal dissection. 
(11) Hemoglobin count $<10 \mathrm{~g} / \mathrm{dL}$; and

(12) Platelet count $<100,000 / \mu \mathrm{L}$

All patients provided informed consent for the procedure. All procedures were conducted in accordance with the ethical standards of the Hospital Ethics Committee and Institutional Review Board of CHA University (approval number: CHAMC 2017-05-033).

\section{ESD procedure}

ESD was performed using an insulation-tipped diathermic knife (IT Knife, KD-610L; Olympus, Tokyo, Japan) and a dual knife. A VIO 300D device (ERBE, Tübingen, Germany) was used as an electrosurgical unit. A circumferential incision was made using the Endocut Q mode (effect 3, cutting duration 3 , cutting interval 2). Hemostasis was performed using the SoftCoag mode (effect 6, 60 watts). Complete removal of the lesion was achieved with submucosal dissection using the swift coagulation mode (effect 3,30 watts). The procedure duration and the number of preventive hemostasis application events, bleeding events, and perforations were recorded. After the procedure, coagulation was performed using Coagrasper forceps (FD-410LR; Olympus) or hemostatic clips (EZ-CLIP, HX-110QR; Olympus). All ESD procedures were performed by a single experienced endoscopist (JYC), who has conducted more than 400 endoscopic resections annually. JYC also performed all follow-up endoscopies.

\section{Study measurements}

The primary endpoint of this study was the assessment of the ulcer size and stage (Sakita classification) after endoscopic resection (28 days after endoscopic surgery), which was reported by the endoscopist who performed the ESD. We conducted a follow-up endoscopy to compare the ulcer stage and ulcer reduction rate or ratio (calculated by dividing the ulcer dimensions at 28 days after ESD by the ulcer dimensions at 24 hours after ESD) between the two groups.

Secondary endpoints included assessment of the rate of acute complications by evaluating the pain score, bleeding, perforation, and stricture after the procedure. The pain score was evaluated using the Numeric Pain Rating Scale (NRS), with a level $>3$ qualifying as pain. Post-ESD bleeding was defined as hemorrhage that persisted for more than 1 minute after ESD with symptoms, including included dizziness, black stool, blood loss, decrease in hemoglobin levels (by $2 \mathrm{~g} / \mathrm{dL}$ or more), or a decrease in blood pressure. Post-ESD perforation was diagnosed endoscopically and/or by the presence of free air on plain radiography and/or computed tomography immediately after ESD. The presence of strictures was evaluated by symptoms (dysphagia, nausea, and vomiting) and endoscopic findings.

\section{Statistical analysis}

The ulcer size and stage were compared between the study and control groups using an independent $t$-test, Fisher's exact test, and the Mann-Whitney $U$ test. The frequency of postoperative complications, such as bleeding, perforation, and delayed stenosis, was analyzed. Differences were considered statistically significant when $p<0.05$.

\section{RESULTS}

In total, 14 patients were included in the study, after 4 patients were excluded for their history of esophageal and stomach surgery and taking NSAIDs within 48 hours before the procedure. We enrolled 7 patients in the study group and 7 patients in the control group. Both groups were similar in terms of sex, age, past medical histories, indications for ESD, and location of the target lesion (Table 1).

In the study group, precancerous lesions such as carcinoma or adenoma in the stomach were included. Final histopathologic findings varied from dysplasia to carcinoma in both groups. There were 3 cases of low-grade dysplasia (LGD), 1 case of high-grade dysplasia, and 3 cases with EGC in the study group. Most of the patients in the control group had LGD (6 patients) and 1 had EGC.

In the study group, the reduction in mean ulcer size 28 days after ESD was $59.71 \%$, compared to $52.57 \%$ in the control group ( $p=0.372$ ). Mean ulcer size reduction was higher in the study group, but the difference was not statistically significant (Table 2) (Fig. 3).

However, 28 days after ESD, there were 5 patients in the $\mathrm{S}$ stage and 2 patients in the $\mathrm{H}$ stage in the study group compared to no patient in the $\mathrm{S}$ stage and 7 patients in $\mathrm{H}$ stage in the control group ( $p=0.05$ ) (Table 3). Scar formation after ESD was more rapid in the study group.

There were no life-threatening serious complications in either group. Two patients in the study group developed bleeding that required blood transfusions, but no one in the control group required a transfusion. Two patients were treated locally with argon plasma coagulation, due to minimal oozing at the resection site, although there was no definitive bleeding after ESD. Three patients in both groups complained of pain after ESD, ranging from 4-5 points on the NRS. There were no cases of perforation or stricture in either group.

\section{DISCUSSION}

Recently, ESD, which enables the en-bloc resection of lesions in the gastrointestinal tract with minimal invasion, ${ }^{27,28}$ 
has gained popularity. However, iatrogenic ulcers and complications, such as significant bleeding, abdominal pain, and delayed wound-healing, are occasionally observed after resection. ${ }^{3}$ Although the mechanism for ESD-induced ulcer healing is unclear, many studies have been conducted to prevent and treat these complications, but no definitive treatment has been established to date. Antiplatelet drugs, such as aspirin and NSAIDs, which inhibit platelet function, interfere with gastric ulcer healing and hemostasis. Since angiogenesis is involved in wound healing, we were interested in the role of platelets in modulating gastric ulcer healing. ${ }^{29}$ During tissue damage, platelets aggregate to induce vascular repair. Pro-angiogenic factors, such as VEGF, fibroblast growth factor, epi- dermal growth factor, and PDGF, stored in the platelets, are then released and regulate wound healing by interacting with anti-angiogenic factors, such as endostatin. ${ }^{30-33}$ Based on this mechanism, PRP containing large amounts of platelets was used in this study for patients who underwent ESD. Additionally, PRP has already been used in various medical disciplines, such as dermatology (for acute and chronic ulcers, such as chronic refractory diabetic ulcer and venous leg ulcers), orthopedics (for muscle injury, ligament injury, tendinopathy, and other such conditions), ${ }^{16-25}$ neurology, ${ }^{34}$ ophthalmology, ${ }^{35}$ and dentistry. ${ }^{36} \mathrm{~A}$ meta-analysis on the use of PRP in experimentally-induced skin wounds with an animal model ${ }^{37}$ and some animal studies on the efficacy and safety of PRPs in

Table 1. Baseline Characteristics of Patients

\begin{tabular}{|c|c|c|c|}
\hline & Control group $(n=7)$ & Study group $(n=7)$ & $p$-value \\
\hline Sex, M:F & $5: 2$ & $7: 0$ & $0.462^{\mathrm{a})}$ \\
\hline Age, yr & $72.57 \pm 7.74$ & $71.57 \pm 5.41$ & $0.784^{\mathrm{b})}$ \\
\hline Procedure time, $\min$ & $37.14 \pm 24.47$ & $42.14 \pm 25.97$ & $0.717^{\mathrm{b})}$ \\
\hline Location of target lesion, $n$ & & & $0.842^{\mathrm{c})}$ \\
\hline Antrum & 3 & 4 & \\
\hline Angle & 1 & 1 & \\
\hline Body & 3 & 2 & \\
\hline Final diagnosis, $n$ & & & $0.223^{\mathrm{c})}$ \\
\hline LGD & 6 & 3 & \\
\hline HGD & 0 & 1 & \\
\hline EGC & 1 & 3 & \\
\hline Complication, $n$ & & & $0.306^{c)}$ \\
\hline None & 5 & 2 & \\
\hline Abdominal pain & 1 & 2 & \\
\hline Major bleeding (required transfusion) & 0 & 2 & \\
\hline Perforation & 0 & 0 & \\
\hline Stenosis & 0 & 0 & \\
\hline Minor bleeding & 1 & 1 & \\
\hline
\end{tabular}

Values expressed as median \pm standard deviation.

EGC, early gastric cancer; HGD, high-grade dysplasia; LGD, low-grade dysplasia.

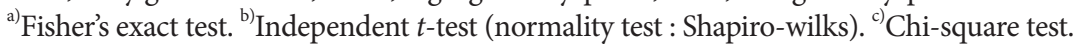

Table 2. Ulcer Size after Endoscopic Submucosal Dissection

\begin{tabular}{lccc}
\hline & Control group $(\boldsymbol{n}=7)$ & Study group $(\boldsymbol{n}=7)$ & $\boldsymbol{p}^{- \text {-value }}$ \\
\hline Ulcer size immediately after ESD, mm & $40.57 \pm 7.41$ & $32.43 \pm 7.39$ & $0.062^{\mathrm{a})}$ \\
Ulcer size after 1 mo after ESD, mm & $16.00 \pm 8.16$ & $12.71 \pm 3.86$ & $0.209^{\text {b) }}$ \\
Reduction rate of ulcer after 1 mo of ESD, mm & $52.57 \pm 15.91$ & $59.71 \pm 12.71$ & $0.372^{\mathrm{a})}$ \\
\hline
\end{tabular}

Values expressed as median \pm standard deviation.

ESD, endoscopic submucosal dissection.

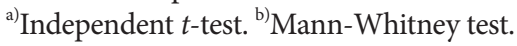


ESD-induced ulcer healing have been published ${ }^{38}$ but PRPs have rarely been used in human studies. PRP is a minimally-processed autologous blood product obtained from one's own body. ${ }^{39}$ A major advantage of PRP is that it can be prepared by centrifugation of the patient's own blood; thus, it is safe, cost-effective, and simple. ${ }^{40,41}$ PRP can be prepared at the patient's bedside and administered immediately. The application of PRP can vary depending on the disease condition (such as in the liquid or gel form for wounds, ${ }^{42}$ shielding with spray for colon ESD in animal models, ${ }^{38}$ submucosal injection for orthodontic purposes, ${ }^{43}$ subcutaneous injection or topical application for non-healing ulcers ${ }^{25}$ ); however, there is no report, which identifies the best method for PRP administration. We used submucosal injections of PRP, as we believed this might prolong the beneficial effects and accelerate ulcer healing. However, additional studies using alternative methods are needed, and further research on the differences of each method is also required.

In our study, local PRP injection at the resection site after ESD was easy and safe, without any serious complications. There was some slight submucosal bleeding during the local injection of PRPs, but this did not require any intervention. Although the difference in the mean reduction of ulcer size was not statistically significant between the two groups, scar formation was significantly faster in the study group. Thus, the local injection of PRP could be a safe and effective method for ulcer healing after endoscopic resection. This is the first human study to show the effect of PRP on ulcer healing post ESD.
There are some limitations in our study: (1) the ulcer size was indirectly measured using an endoscopic probe. In the control group, the size of the ulcer after resection of the lesion was approximately $1 \mathrm{~cm}$, which probably influenced the healing rate. Although patients with ulcer size $>2 \mathrm{~cm}$ were not enrolled in this study, the PRP effect was more pronounced in the study group than in the control group. Larger lesion sizes will be studied in a follow-up study; (2) intravenous and oral PPI therapy were administered in all cases; hence, it was difficult to clearly identify, the independent effect of PRP. Therefore, a study showing the effect of a local injection of PRP without intravenous PPI therapy is necessary; (3) our study had a small patient population ( $n=7$ in each group); (4) a continuous long-term follow-up is necessary, since our study only had a 28-day follow-up period; and (5) although PRP has the advantage of being an autologous product extracted from the patient's own blood, the preparation protocol after analyzing the PRP components and practical methods for clinical use are still unclear. The various factors associated with PRP should be analyzed and the components should be sampled to develop practical methods for PRP preparation and administration for clinical use.

In conclusion, local injection of PRP is safe and easy, and this is a promising technique for preventing post-ESD wound complications. A larger sample size and additional long-term follow-up studies are needed to establish the efficacy and safety of local PRP injection for post-ESD wound healing.
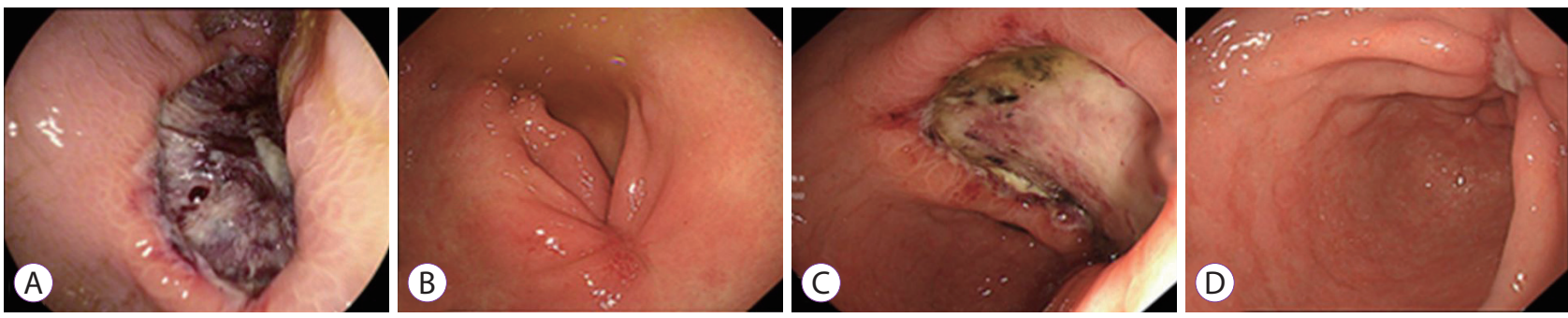

Fig. 3. Change of ulcer (endoscopic finding) 24 hours after endoscopic submucosal dissection.(A) Study (platelet-rich plasma) group 24 hours after endoscopic submucosal dissection (ESD). (B) Study (platelet-rich plasma) group 28 days after ESD. (C) Control group 24 hours after ESD. (D) Control group 28 days after ESD.

Table 3. Ulcer Stage at 28 Days after Endoscopic Submucosal Dissection

\begin{tabular}{lccc}
\hline & Control group $(\boldsymbol{n}=7)$ & Study group $(\boldsymbol{n}=\mathbf{8})$ & $\boldsymbol{p}_{\text {-value }}$ \\
\hline $\mathrm{H} 1, n$ & 3 & 1 & $0.05^{\mathrm{a})}$ \\
$\mathrm{H} 2, n$ & 4 & 1 & \\
$\mathrm{~S} 1, n$ & 0 & 3 & \\
$\mathrm{~S} 2, n$ & 0 & 2 & \\
\hline
\end{tabular}

${ }^{\text {a) }}$ Fisher's exact test. 
Conflicts of Interest

The authors have no financial conflicts of interest.

\section{Acknowledgements}

We would like to thank Editage (www.editage.co.kr) for English language editing.

\section{REFERENCES}

1. Kim DS, Jung Y, Rhee HS, et al. Usefulness of the forrest classification to predict artificial ulcer rebleeding during second-look endoscopy after endoscopic submucosal dissection. Clin Endosc 2016;49:273-281.

2. Oda I, Nonaka S, Abe S, Suzuki H, Yoshinaga S, Saito Y. Is there a need to shield ulcers after endoscopic submucosal dissection in the gastrointestinal tract? Endosc Int Open 2015;3:E152-E153.

3. Ono H, Kondo H, Gotoda T, et al. Endoscopic mucosal resection for treatment of early gastric cancer. Gut 2001;48:225-229.

4. Lee SY, Kim JJ, Lee JH, et al. Healing rate of EMR-induced ulcer in relation to the duration of treatment with omeprazole. Gastrointest Endosc 2004;60:213-217.

5. Kakushima N, Yahagi N, Fujishiro M, et al. The healing process of gastric artificial ulcers after endoscopic submucosal dissection. Dig Endosc 2004;16:327-331.

6. Park JH, Baek EK, Choi CH, et al. Comparison of the efficacy of 4- and 8-week lansoprazole treatment for ESD-induced gastric ulcers: a randomized, prospective, controlled study. Surg Endosc 2014;28:235-241.

7. Ye $\mathrm{BD}$, Cheon JH, Choi KD, et al. Omeprazole may be superior to famotidine in the management of iatrogenic ulcer after endoscopic mucosal resection: a prospective randomized controlled trial. Aliment Pharmacol Ther 2006;24:837-843.

8. Takao T, Takegawa Y, Shinya N, Tsudomi K, Oka S, Ono H. Tissue shielding with polyglycolic acid sheets and fibrin glue on ulcers induced by endoscopic submucosal dissection in a porcine model. Endosc Int Open 2015;3:E146-E151.

9. Takimoto K, Toyonaga T, Matsuyama K. Endoscopic tissue shielding to prevent delayed perforation associated with endoscopic submucosal dissection for duodenal neoplasms. Endoscopy 2012;44 Suppl 2 UCT$\mathrm{N}: \mathrm{E} 414-\mathrm{E} 415$

10. Takimoto K, Imai Y, Matsuyama K. Endoscopic tissue shielding method with polyglycolic acid sheets and fibrin glue to prevent delayed perforation after duodenal endoscopic submucosal dissection. Dig Endosc 2014;26 Suppl 2:46-49.

11. Marx RE. Platelet-rich plasma (PRP): what is PRP and what is not PRP? Implant Dent 2001;10:225-228.

12. Molloy T, Wang Y, Murrell G. The roles of growth factors in tendon and ligament healing. Sports Med 2003;33:381-394.

13. Akimoto M, Hashimoto H, Maeda A, Shigemoto M, Yamashita K. Roles of angiogenic factors and endothelin-1 in gastric ulcer healing. Clin Sci (Lond) 2002;103 Suppl 48:450S-454S.

14. Luo JC, Shin VY, Liu ES, et al. Dexamethasone delays ulcer healing by inhibition of angiogenesis in rat stomachs. Eur J Pharmacol 2004; 485:275-281.

15. Perini R, Wallace JL, Ma L. Roles of platelets and proteinase-activated receptors in gastric ulcer healing. Dig Dis Sci 2005;50 Suppl 1:S12-S15.

16. A Hamid MS, Mohamed Ali MR, Yusof A, George J, Lee LP. Platelet-rich plasma injections for the treatment of hamstring injuries: a randomized controlled trial. Am J Sports Med 2014;42:2410-2418.

17. Carter MJ, Fylling CP, Parnell LK. Use of platelet rich plasma gel on wound healing: a systematic review and meta-analysis. Eplasty 2011;11:e38.

18. Chicharro-Alcántara D, Rubio-Zaragoza M, Damiá-Giménez E, et al. Platelet rich plasma: new insights for cutaneous wound healing man- agement. J Funct Biomater 2018;9:E10.

19. Hammond JW, Hinton RY, Curl LA, Muriel JM, Lovering RM. Use of autologous platelet-rich plasma to treat muscle strain injuries. Am J Sports Med 2009;37:1135-1142.

20. Kazakos K, Lyras DN, Verettas D, Tilkeridis K, Tryfonidis M. The use of autologous PRP gel as an aid in the management of acute trauma wounds. Injury 2009;40:801-805.

21. Lacci KM, Dardik A. Platelet-rich plasma: support for its use in wound healing. Yale J Biol Med 2010;83:1-9.

22. Mehrannia M, Vaezi M, Yousefshahi F, Rouhipour N. Platelet rich plasma for treatment of nonhealing diabetic foot ulcers: a case report. Can J Diabetes 2014;38:5-8.

23. Picard F, Hersant B, Bosc R, Meningaud JP. Should we use platelet-rich plasma as an adjunct therapy to treat "acute wounds," "burns," and "laser therapies": a review and a proposal of a quality criteria checklist for further studies. Wound Repair Regen 2015;23:163-170.

24. Samani MK, Saberi BV, Ali Tabatabaei SM, Moghadam MG. The clinical evaluation of platelet-rich plasma on free gingival graft's donor site wound healing. Eur J Dent 2017;11:447-454.

25. Suthar M, Gupta S, Bukhari S, Ponemone V. Treatment of chronic non-healing ulcers using autologous platelet rich plasma: a case series. J Biomed Sci 2017;24:16.

26. Sakita T, Fukutomi H. Endoscopic diagnosis. In: Yoshitoshi Y, ed. Ulcer of stomach and duodenum. Tokyo: Nankodo; 1971. p. 198-208.

27. Baniya R, Upadhaya S, Khan J, et al. Carbon dioxide versus air insufflation in gastric endoscopic submucosal dissection: a systematic review and meta-analysis of randomized controlled trials. Clin Endosc 2017;50:464-472.

28. Lee HJ, Lee YJ, Lee JY, et al. Characteristics of synchronous and metachronous multiple gastric tumors after endoscopic submucosal dissection of early gastric neoplasm. Clin Endosc 2018;51:266-273.

29. Ma L, Elliott SN, Cirino G, Buret A, Ignarro LJ, Wallace JL. Platelets modulate gastric ulcer healing: role of endostatin and vascular endothelial growth factor release. Proc Natl Acad Sci U S A 2001;98:6470-6475.

30. Hwang DL, Lev-Ran A, Yen CF, Sniecinski I. Release of different fractions of epidermal growth factor from human platelets in vitro: preferential release of $140 \mathrm{kDa}$ fraction. Regul Pept 1992;37:95-100.

31. O'Reilly MS, Boehm T, Shing Y, et al. Endostatin: an endogenous inhibitor of angiogenesis and tumor growth. Cell 1997;88:277-285.

32. Wartiovaara U, Salven P, Mikkola H, et al. Peripheral blood platelets express VEGF-C and VEGF which are released during platelet activation. Thromb Haemost 1998;80:171-175.

33. Yamaguchi N, Anand-Apte B, Lee M, et al. Endostatin inhibits VEGF-induced endothelial cell migration and tumor growth independently of zinc binding. EMBO J 1999;18:4414-4423.

34. Salarinia R, Sadeghnia HR, Alamdari DH, Hoseini SJ, Mafinezhad A, Hosseini M. Platelet rich plasma: effective treatment for repairing of spinal cord injury in rat. Acta Orthop Traumatol Turc 2017;51:254-257.

35. Ronci C, Ferraro AS, Lanti A, et al. Platelet-rich plasma as treatment for persistent ocular epithelial defects. Transfus Apher Sci 2015;52:300-304.

36. Ghoddusi J, Maghsudlu A, Jafarzadeh H, Jafarian A, Forghani M. Histological evaluation of the effect of platelet-rich plasma on pulp regeneration in nonvital open apex teeth: an animal study. J Contemp Dent Pract 2017;18:1045-1050.

37. Tambella AM, Attili AR, Dupré G, et al. Platelet-rich plasma to treat experimentally-induced skin wounds in animals: a systematic review and meta-analysis. PLoS One 2018;13:e0191093.

38. Lorenzo-Zúñiga V, Boix J, Moreno de Vega V, Bon I, Marín I, Bartolí R. Efficacy of platelet-rich plasma as a shielding technique after endoscopic mucosal resection in rat and porcine models. Endosc Int Open 2016;4:E859-E864.

39. Chahla J, Cinque ME, Piuzzi NS, et al. A call for standardization in platelet-rich plasma preparation protocols and composition reporting: a systematic review of the clinical orthopaedic literature. J Bone Joint Surg Am 2017;99:1769-1779. 


\section{$C_{\text {cunncal ENoscopr }}$}

40. Bernuzzi G, Tardito S, Bussolati O, et al. Platelet gel in the treatment of cutaneous ulcers: the experience of the Immunohaematology and Transfusion Centre of Parma. Blood Transfus 2010;8:237-247.

41. Leitner GC, Gruber R, Neumüller J, et al. Platelet content and growth factor release in platelet-rich plasma: a comparison of four different systems. Vox Sang 2006;91:135-139.

42. Kim SA, Ryu HW, Lee KS, Cho JW. Application of platelet-rich plas- ma accelerates the wound healing process in acute and chronic ulcers through rapid migration and upregulation of cyclin A and CDK4 in HaCaT cells. Mol Med Rep 2013;7:476-480.

43. Liou E. The development of submucosal injection of platelet rich plasma for accelerating orthodontic tooth movement and preserving pressure side alveolar bone. APOS trends in orthodontics 2016;6:5-11. 